\title{
Beta Measurements and Modeling the Tevatron
}

\author{
Norman M. Gelfand \\ Fermi National Accelerator Laboratory \\ P.O. Box 500, Batavia, Illinois 60510
}

June 1993

Presented at the 1993 Particle Accelerator Conference, Washington, D.C., May 17-20, 1993 


\section{Disclaimer}

This report was prepared as an account of work sponsored by an agency of the United States Government. Neither the United States Government nor any agency thereof, nor any of their employees, makes any warranty, express or implied, or assumes any legal liability or responsibility for the accuracy, completeness, or usefulness of any information, apparatus, product, or process disclosed, or represents that its use would not infringe privately owned rights. Reference herein to any specific commercial product, process, or service by trade name, trademark, manufacturer, or otherwise, does not necessarily constitute or imply its endorsement, recommendation, or favoring by the United States Government or any agency thereof. The views and opinions of authors expressed herein do not necessarily state or reflect those of the United States Government or any agency thereof. 


\title{
Beta Measurements And Modeling The Tevatron \\ Norman M. Gelfand \\ Fermi National Accelerator Laboratory* \\ P.O. Box 500, Batavia II. 60510, USA
}

\begin{abstract}
The Tevatron collider is now able to operate with two low $\beta\left(\beta^{*}=0.25-0.5 \mathrm{~m}\right)$ interaction regions denoted as $B 0$ and $D 0$. This lattice allows independent operation of the interaction regions which required that the previous collider lattice, used in 1988-89, had to be modified. In order to see how well the lattice conforms to the design, measurements of the $\beta$ function have been carried out at 15 locations in the new Tevatron collider lattice. Agreement can be obtained between the measurements and a computer model for the Tevatron, based on the design, only if the strengths of the gradients in the quadrupoles in the low- $\beta$ triplet are allowed to differ from their design values. It is also observed that the lattice is very sensitive to the precise values of the gradients in these magnets.
\end{abstract}

\section{Introduction}

The Tevatron is able to operate as a collider with two low $\beta\left(\beta^{*}=0.25-0.5 \mathrm{~m}\right)$ interaction regions denoted as $\mathrm{B} 0$ and $\mathrm{D} 0$. This lattice was designed to allow independent operation of the interaction regions and is quite different from the previous collider lattice, used in 1988-89. The design of these new low- $\beta$ insertions required the construction of new, strong, quadrupoles to get to the desired value of $\beta^{*}=0.25 \mathrm{~m}$ at $1 \mathrm{TeV}$. In addition new quadrupole correctors are required outside of the triplet to match the lattice functions of the low- $\beta$ insertion with the values in the lattice arcs at the matching point, an essential requirement if operation of the interaction regions is to be independent.

In addition to a new interaction region at D0, the Tevatron collider closed orbit is a helix produced by electrostatic separators. This is done to reduce the beam-beam interaction between the colliding protons and anti-protons. We can now run with smaller proton emittances, compared to the last collider run, and thus increase the luminosity. Additional separators are used to make the beams collide head on at the interaction points. The use of separated orbits complicates operations but has only a small effect on the lattice functions.

As with any new facility it is very desirable to test how well the actual performance matches the design expectations. With this as our objective, the lattice function $\beta$ was measured at 14 locations within the insertion, and also at one point in the arc, and the results compared to the calculations of $\beta$ based on the design lattice. This note describes the results of that comparison.

\section{Method}

The method used to measure the amplitude function $\beta$ was proposed by Courant and Snyder in their original paper on the Alternate Gradient Synchrotron[1]. The procedure is to vary the strength of a single quadrupole, at a point in the lattice where it is possible to do so, and measure the resulting change in the tunes. The change in tune, in a given plane, is related to the value of $\beta$ in that plane at that point, according to the following formula:

$$
\beta=4 \pi \Delta v /\left(\mathrm{L} \Delta \mathrm{k}^{2}\right)
$$

where $\Delta k^{2}=\Delta B^{\prime} /[B \rho]$ and $\Delta B^{\prime}$ is the change in the gradient of the quadrupole and $L$ is its length.

Because this relation is true only in the limit of very small $\Delta B^{\prime}$, at which the change in tune is not measurable, tune changes were measured over a range of $\Delta \mathrm{B}^{\prime}$ and the data were fit to a second order polynomial. The slope of the fit at $\Delta B^{\prime}=0$ gives the desired value of $\beta$.

The measurements of $\beta$, using this procedure, were made at 7 locations on both sides of the interaction regions at BO and DO and at near the location of a tuning quadrupole at E17. During these measurements there were only protons in the Tevatron.

It is obvious that no measurements, using this approach, can be made at the interaction points since those points must be left clear for the experiments. Further the low value of $\beta$ there

* Operated by Universities Research Association Inc., under contract with the United States Department of Energy. 
means that even if a quadrupole could be placed at the interaction point large changes would have to be made in the gradient to observe any tune shift. Knowledge of the values of the lattice functions at the interaction point must come from a model of the lattice.

\section{Comparison With A Model For The Tevatron}

An accurate model of the Tevatron would be able to reproduce the measurements made of the $\beta$ function described above. As is seen the agreement between the measurements and the model using the design values for the the guadrupole gradients is not good (see figures $1 \mathrm{a}, 1 \mathrm{~b}, 1 \mathrm{c}$ ). The reason for the discrepancy was due to an inadequate knowledge of the transfer functions (the relationship between the current in the magnet and the resulting gradient) of the strong quadrupoles in the magnetic triplet.

The $\beta$ function in the present low- $\beta$ Tevatron lattice is very sensitive to small changes in the strengths of the quadrupoles in the low- $\beta$ triplet. Figure 2 shows the change in the $\beta$ function in the arcs when the gradients of the 232 " quadrupoles at $\mathrm{B} 0$ are changed by $0.1 \%$. In order to predict the behavior of the $\beta$ function in the Tevatron it is necessary to know the transfer constants, i.e. the relationship between the current through the quadrupole and the resulting gradient, for the magnets in the triplet to better than $0.1 \%$.

The fabrication methods used to construct these quadrupoles would lead us to expect magnet to magnet variations in the transfer constant of $0.05 \%$ for the 232 "quadrupoles and $0.07 \%$ for the 132 " quadrupoles. Thus the manufacturing tolerances are not good enough to enable us to predict the characteristics of the lattice. The other new quadrupoles in the low- $\beta$ insertions have larger uncertainties $(\sim 0.1 \%)$ but are they weaker and are also at points in the lattice where they are less critical in determining the values of $\beta$.

An attempt was made to measure the transfer constants at the Fermilab Magnet Test Facility (MTF). Unfortunately there were serious problems with the measuring techniques which made the absolute values of the measured transfer constants unreliable, and therefore they have not been used in our model calculations.

Despite the fact that we could not measure, at MTF, the absolute values of the transfer constants we should be able to make use of the relative strengths of the magnets as measured at MTF to account for the variance in quadrupole gradient due to manufacturing tolerances. These relative measurements have been incorporated into our model.

We have attempted to fit the measured values of $\beta$ by varying, in our computer model, the strengths of the magnets in the triplet. Unfortunately the properties of the triplet are such that the three quadrupoles are at essentially the same phase. This means that we are unable to extract meaningful values only for the strength of the triplet and not for the individual elements.

We find reasonable agreement between the model and the data with reasonable changes in the nominal values of the quadrupoles in the triplet (figures $3 a, 3 b, 3 c$ ). Because of the correlation between the fitted transfer constants this procedure does not yield the values of the transfer constants for the individual quadrupoles but the procedure does give a reasonable model of the lattice.

\section{Conclusions}

The $\beta$ function in the Tevatron collider lattice is very sensitive to the precise values of the strengths of the quadrupoles in the low- $\beta$ triplet. Changes in the integrated gradients by $0.1 \%$ can produce significant changes in $\beta$ in the arcs as well as in the low- $\beta$ insertions.

We have been able to get reasonable agreement between the measured values of $\beta$ and our model by making reasonable changes in the nominal values of the strengths of the magnets in the triplets producing the low- $\beta$ interaction point.

\section{$V$. References}

[1]E. D. Courant and H.S. Snyder, "The Theory of the Alternating Gradient Synchrotron", Ann of Phy Vol 3, No 1,1(1958). 


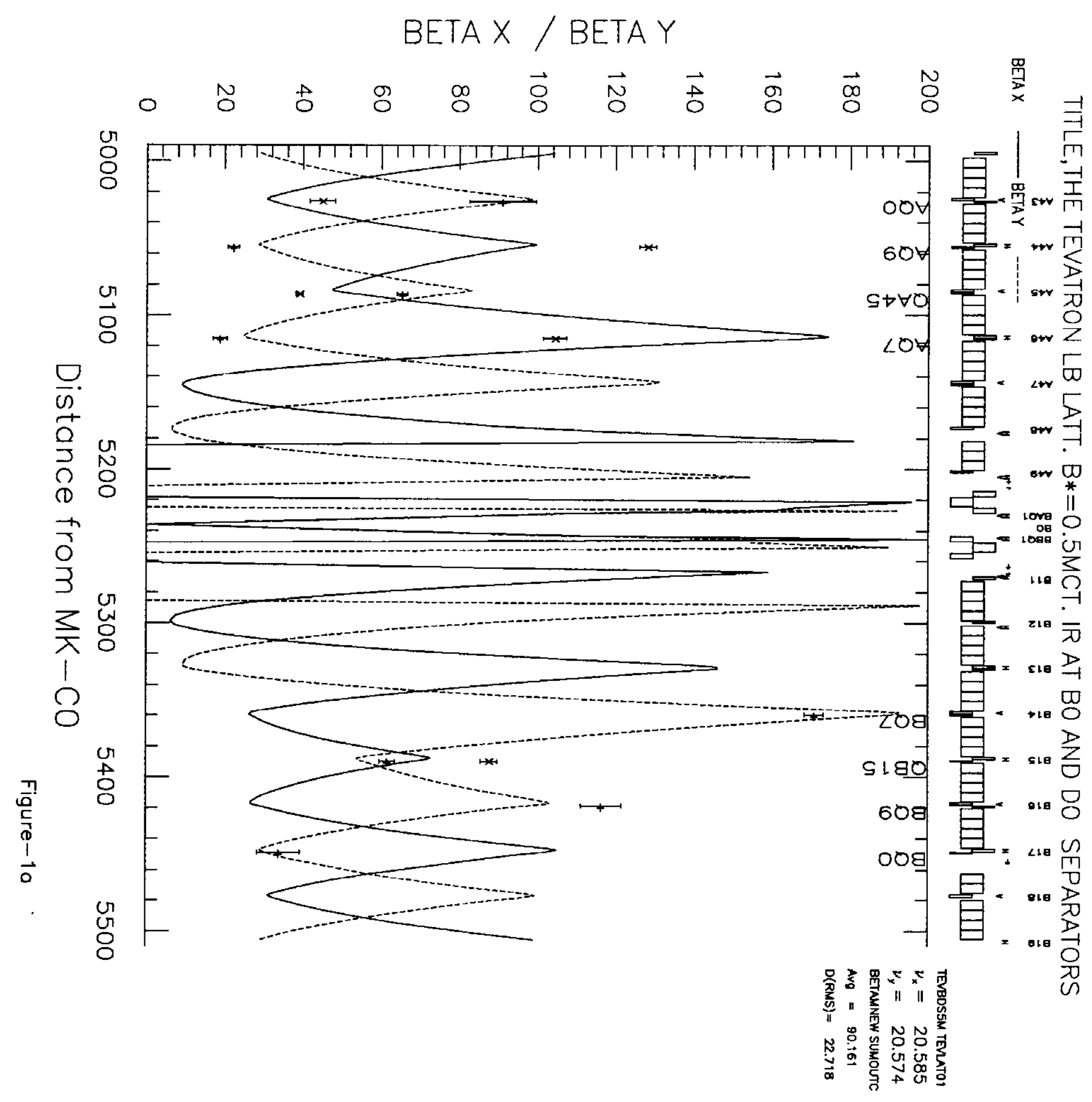


TITLE, THE TEVATRON LB LATT. B* $=0.5 \mathrm{MCT}$. IR AT BO AND DO SEPARATORS

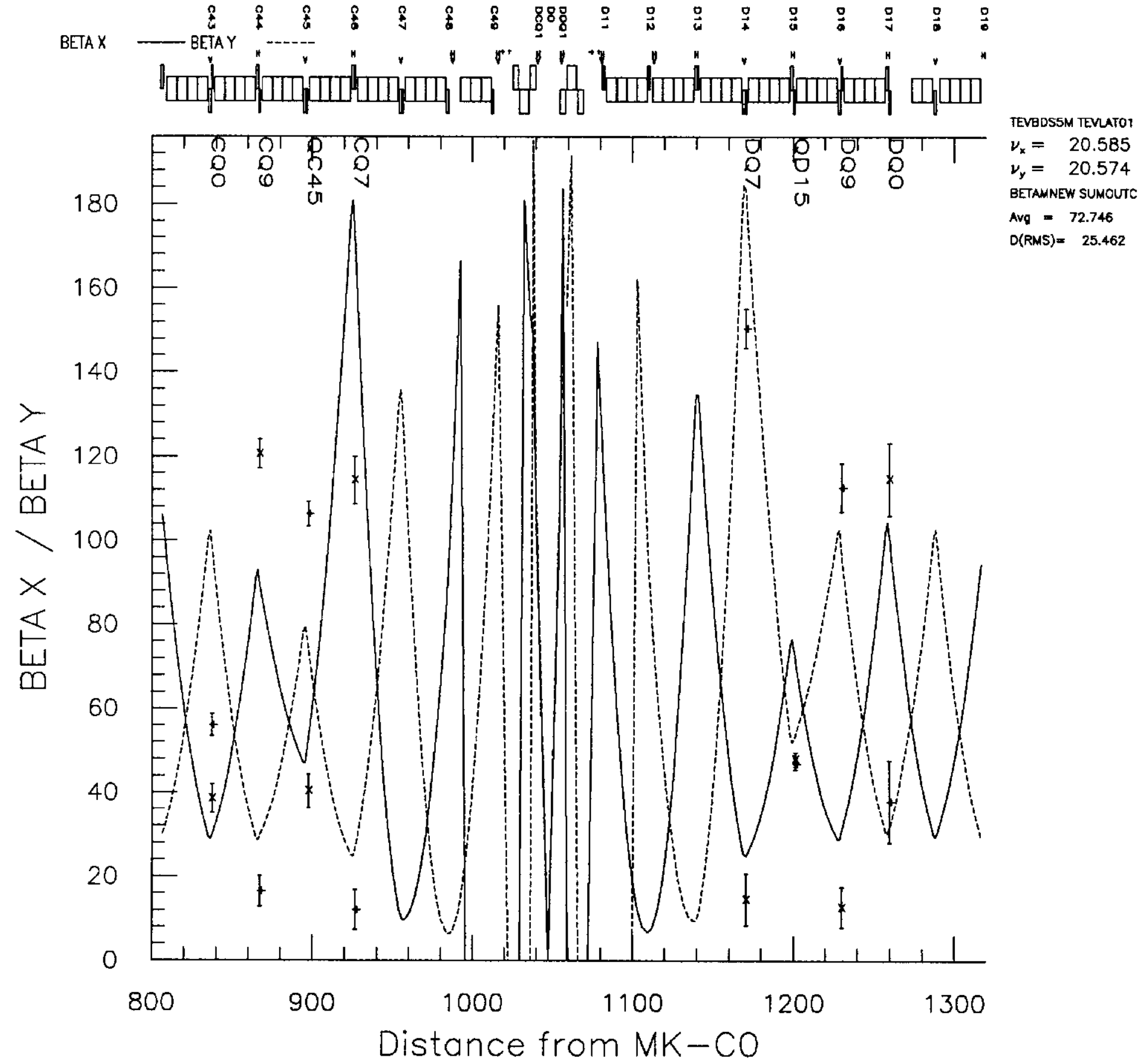

Figure-1b 
TITLE,THE TEVATRON LB LATT. B*=0.5MCT. IR AT BO AND DO SEPARATORS

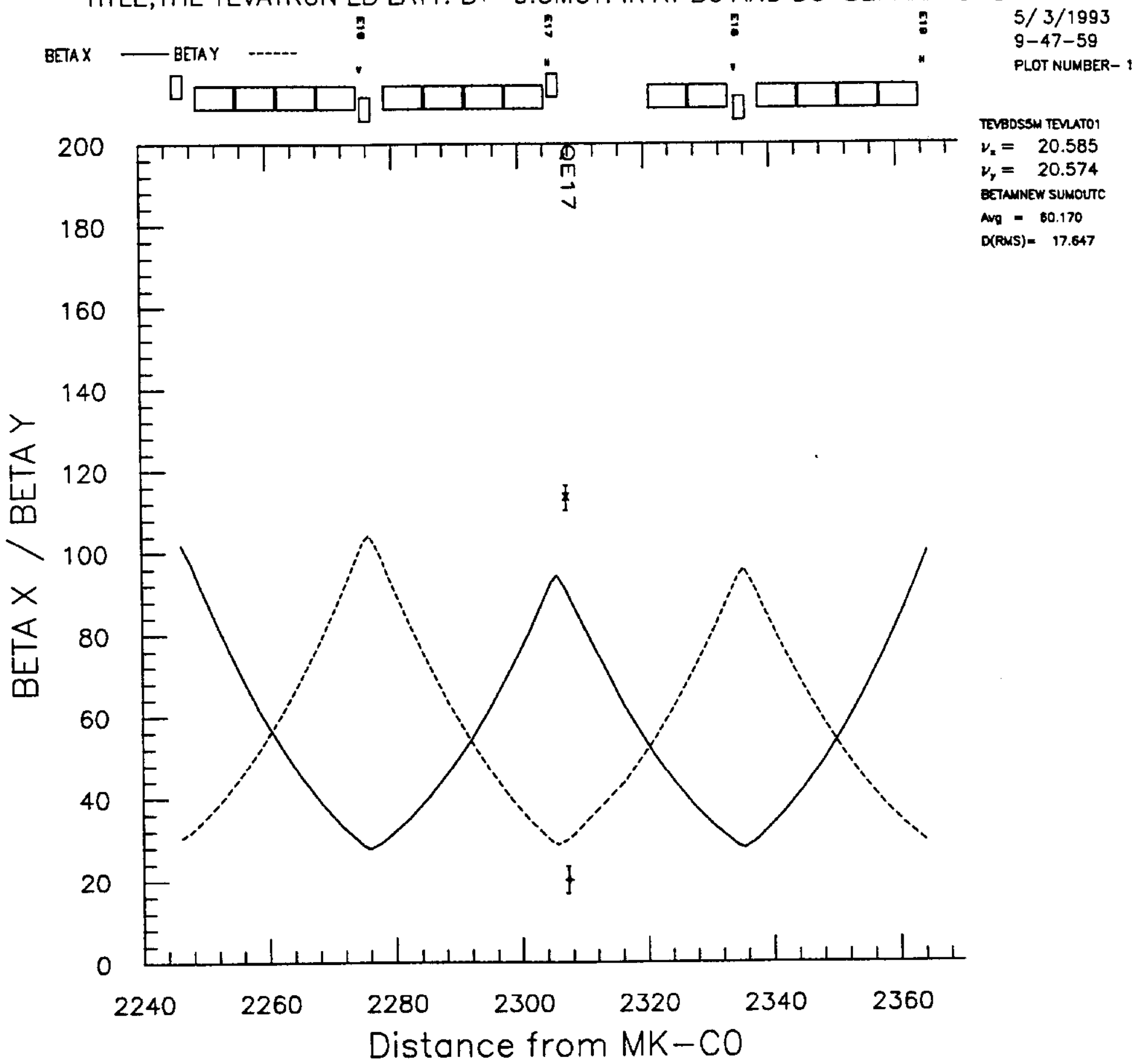

Figure-1c 
TITLE,THE TEVATRON LB LATT. B $*=0.5 \mathrm{MCT}$. IR AT BO AND DO SEPARATORS

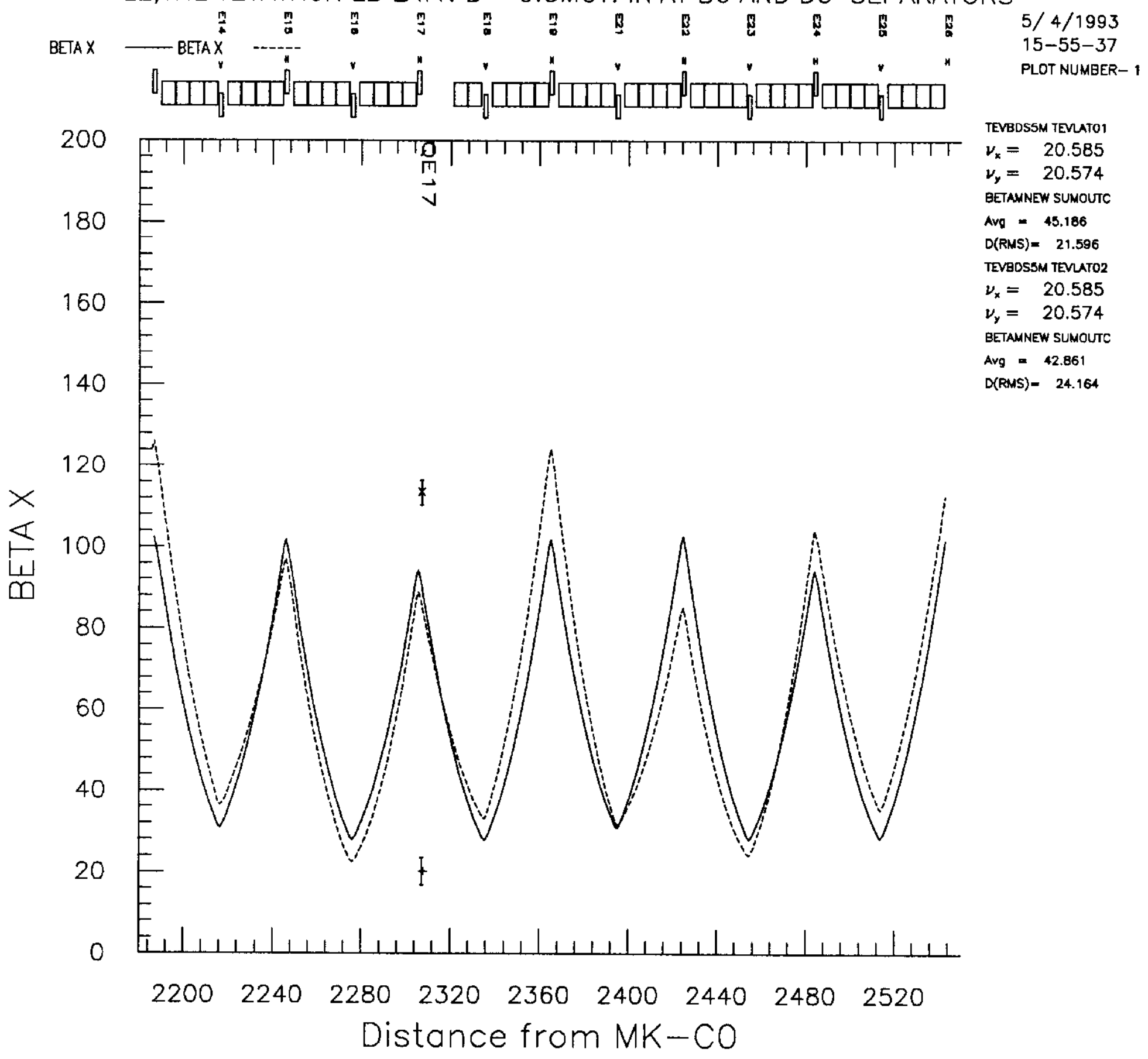

Figure-2 
TITLE, THE TEVATRON LB LATT. B $*=0.5 M C T$. IR AT BO AND DO SEPARATORS

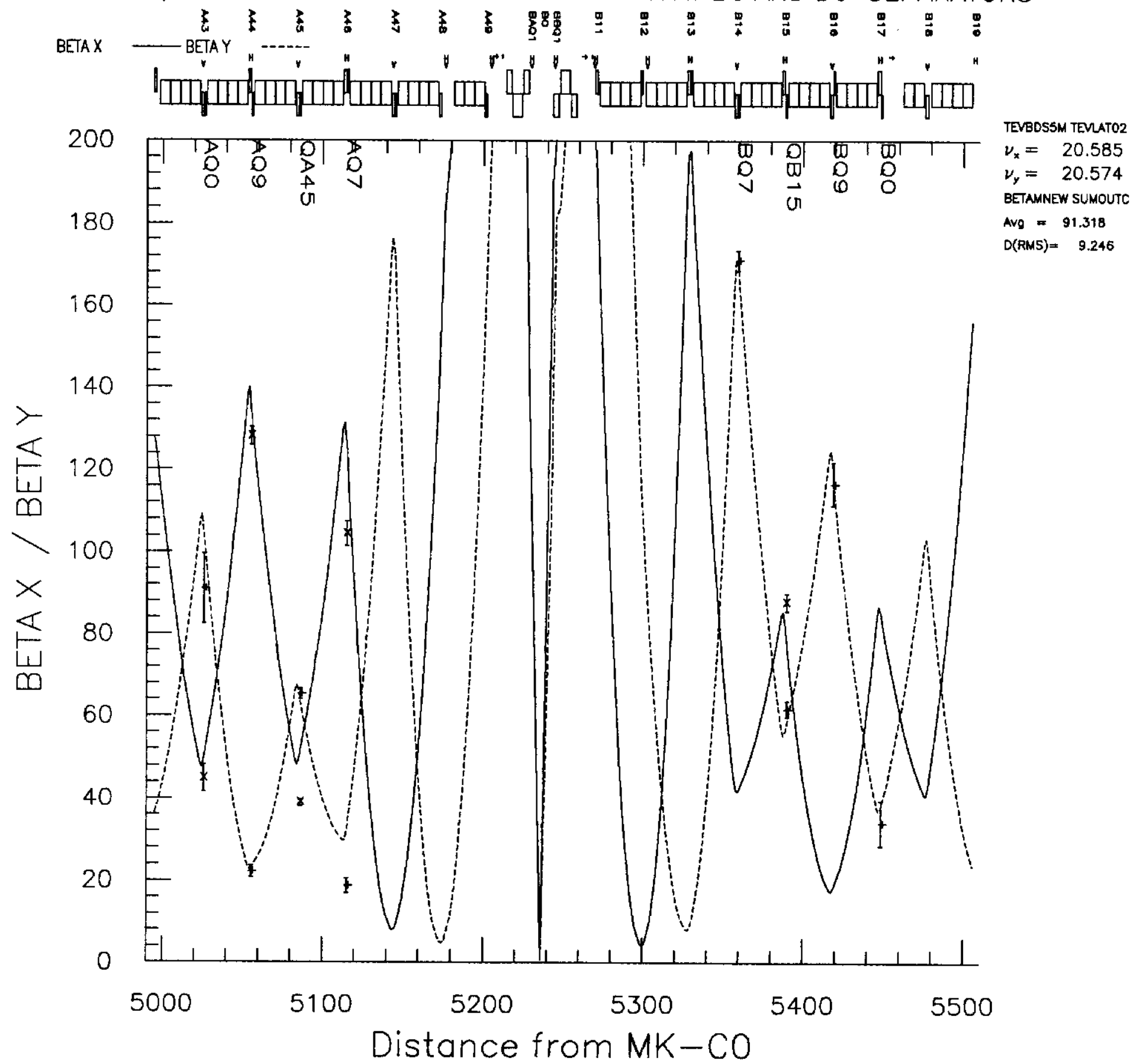

Figure-3a 
TITLE, THE TEVATRON LB LATT. B*=0.5MCT. IR AT BO AND DO SEPARATORS

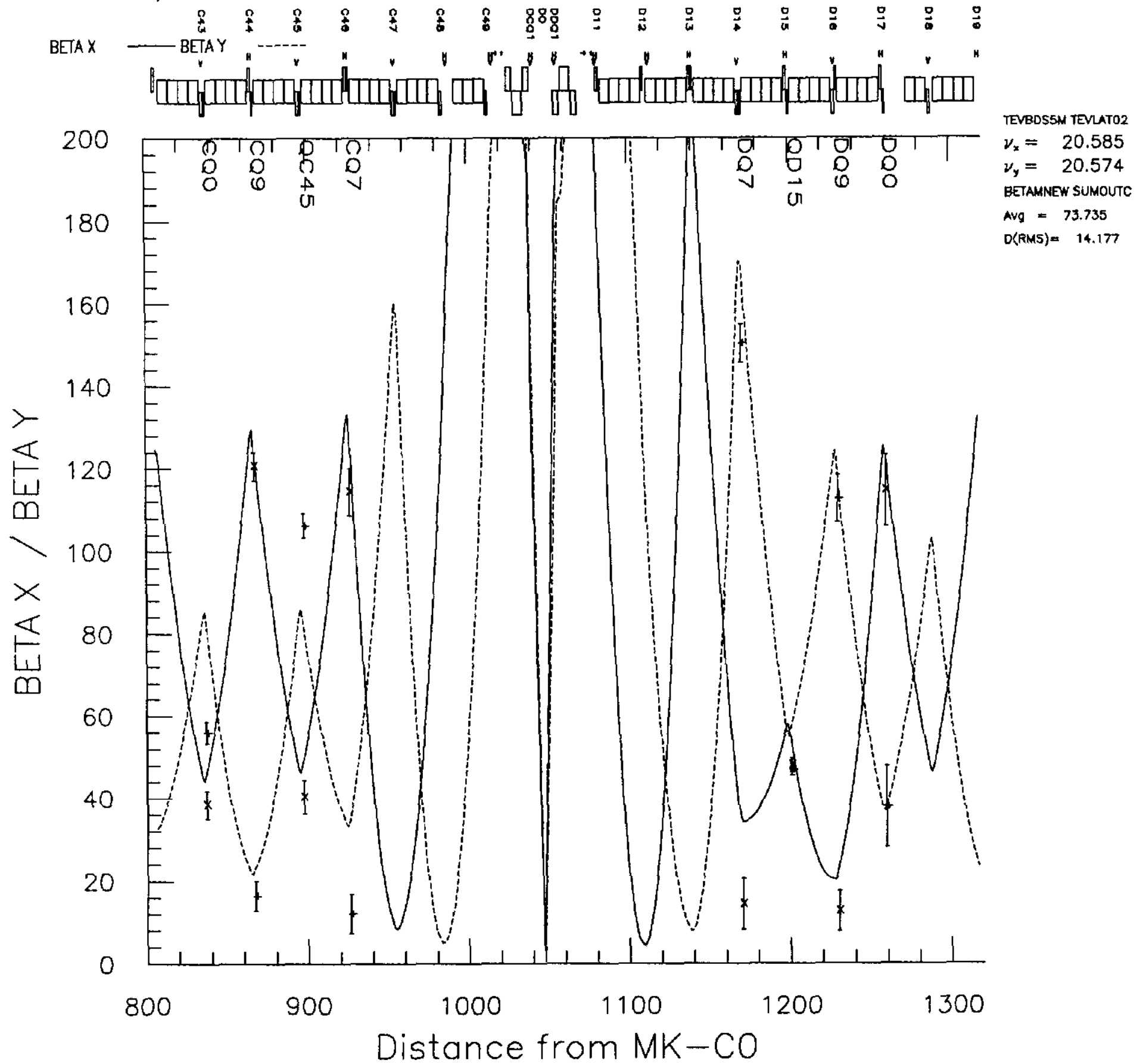

Figure-3b 
TITLE, THE TEVATRON LB LATT. B* $=0.5 \mathrm{MCT}$. IR AT BO AND DO SEPARATORS

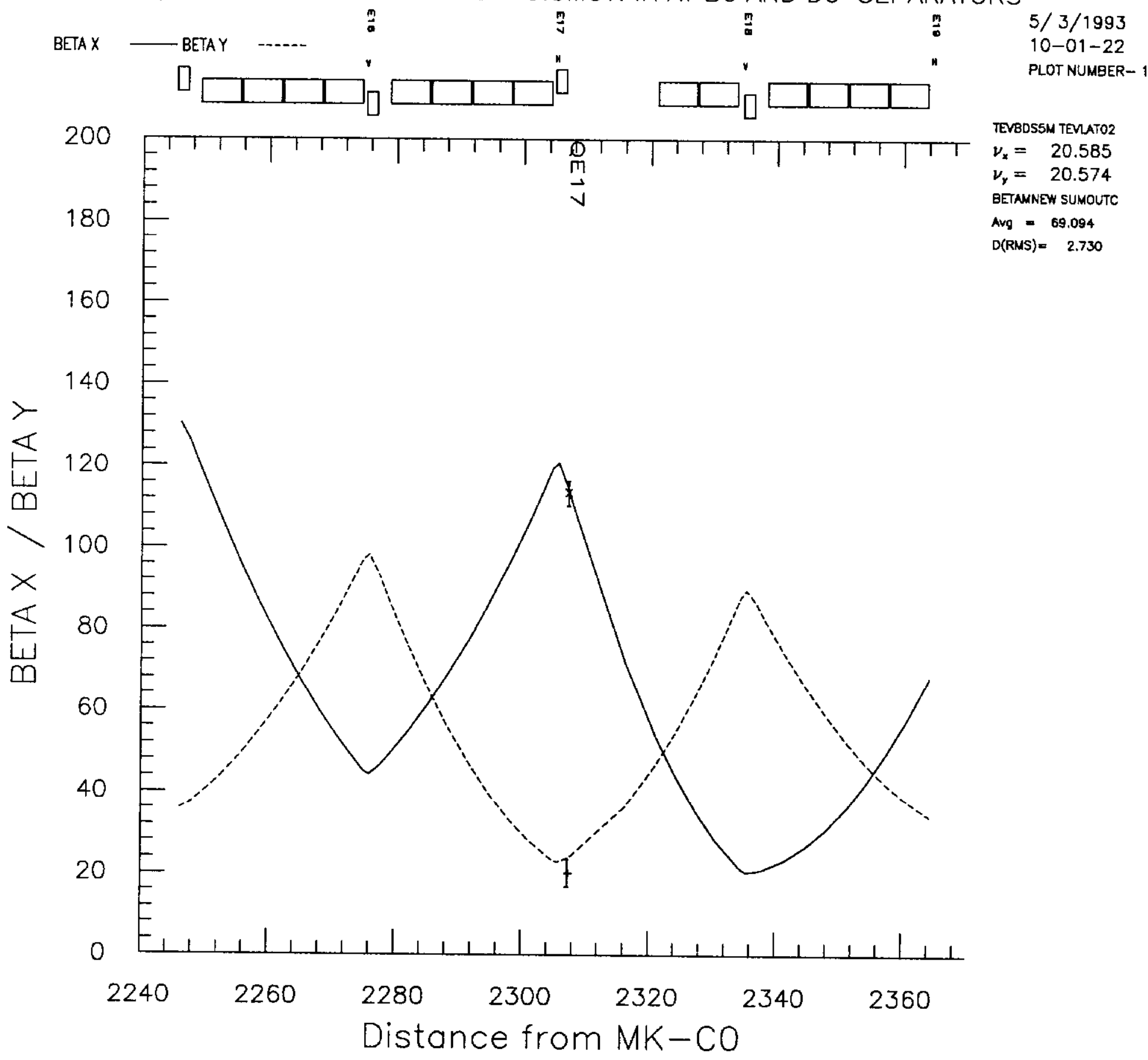

Figure-3C 